\title{
Accurate measurement of stiffness of leukemia cells and leukocytes using optical trap by rate-jump method
}

\author{
Z.L. Zhou ${ }^{1}$, T.H. Hui ${ }^{1}$, B Tang ${ }^{2, *}$ and A.H.W. Ngan ${ }^{1, *}$ \\ ${ }^{1}$ Department of Mechanical Engineering, The University of Hong Kong, Hong Kong \\ ${ }^{2}$ Department of Materials Science and Engineering, South University of Science and \\ Technology of China, Shenzhen, China \\ *Correspondence authors: hwngan@hku.hk / tangbinsci@gmail.com
}

\begin{abstract}
Accurate measurement of the elastic modulus of soft biological cells in the micro/nano scale range is still a challenging task. Tests involving constant-rate loading often yield results which are rate dependent, due to the viscous component of the deformation. In this work, a rate-jump indentation method was employed on an optical tweezers system to measure the stiffness of non-adherent blood cells, which are the softest types of cells. Compared to the traditional Hertzian method of indentation, the rate-jump method is found to be able to yield invariant elastic modulus from K562 myelogenous leukemia cells. The optical tweezers indentation method proposed can therefore serve as a standard protocol for obtaining the intrinsic elastic modulus of extremely soft cells, with applied forces in the pico-newton range. This method is also found to be effective in grading the stiffness values of myelogenous leukemia cell lines (K562 and HL60) and normal leukocytes, indicating that it can be used to identify normal cells from diseased counterparts without biochemical analysis.
\end{abstract}

Keywords: Optical tweezers; Rate-jump tests; Elastic Modulus; Leukocytes; 
Leukemia 


\section{Introduction}

Accumulating evidences have shown that the stiffness of cells is closely related to their death ${ }^{1}$, migration ${ }^{2}$ and differentiation ${ }^{3}$. Atomic force microscope indentation 4,5 , micropipette aspiration ${ }^{6}$ and magnetic tweezers ${ }^{7}$ are commonly used methods to probe the stiffness of cells. However, a cell comprises both viscous and elastic components ${ }^{8,9}$, and so the overall force-displacement response represents the combined effects of both components, no matter whether the experimental platform is indentation, suction or traction $4,7,10,11$. We have recently proven that, for a viscoelastic material governed by a constitutive law in which linear elastic springs are connected to (in general) non-linear viscous dashpots forming any network arrangement, the response during a sudden jump in the loading or straining rate is governed only by that of the elastic springs, while the dashpots behave completely rigidly ${ }^{12,13}$. This provides a means to measure the intrinsic elastic modulus of a viscoelastic material in any test platform. For example, in a tensile test, if the loading rate undergoes a step jump at time $t_{c}$, then the intrinsic Young's modulus at $t_{c}$ is given as the ratio of the difference in stress rates to the difference in strain rates across the jump ${ }^{14}$. If the material behavior is time- or structure-dependent, then the modulus measured this way will be specific to the moment at which the rate jump is imposed, but will be independent of magnitude of the rate jump at that time point, i.e. it will be an intrinsic material property ${ }^{12-14}$. This method is better than the traditional viscoelasticity analysis of the overall force-displacement response which would require the assumption of linear viscous behavior and an a priori known spring-dashpot network as the constitutive law ${ }^{15-19}$. However, the rate-jump method provides no information on the dashpots or viscous components of the constitutive law.

In this paper, we demonstrate that the intrinsic elastic moduli of living cells can be extracted by using optical tweezers based on the rate-jump method. In contrast with other methods including atomic force microscopy (AFM), micropipette 
aspiration and magnetic tweezers, carrying out lateral indentation in an optical tweezers has the merit of allowing the indentation processing to be recorded, thus offering control of the indenter sphere at the desired indentation position with submicron precision ${ }^{20-22}$.

Whether leukocytes or leukemia cells are stiffer has been a long-term debate. The crux of the controversy has been the large scatter often seen in the reported values of cell stiffness ${ }^{23}$, and such scatter is primarily due to the viscous effects of the cell structures and variations in the cell geometry, both of which are factors that can significantly affect the elastic modulus measurement $4,12,24$. On the other hand, reliable measurement of cell stiffness may be helpful in offering quantitative information for certain diseases ${ }^{1,20,25-28}$. In this report, the myelogenous leukemia cell line K562 ${ }^{29}$ was used as a prototype sample for lateral indentation experiments by polystyrene microspheres trapped by optical tweezers, with a purpose to evaluate the advantage of using the rate-jump protocol over the commonly used Hertzian method ${ }^{30}$. We also compared the stiffness of normal leukocytes with myeloid leukemia cells, and observed a higher stiffness value in the leukemia cells. This shows that accurately measured cell stiffness can be a good indicator to differentiate between normal and diseased cells.

\section{Materials and Methods}

\subsection{Cell culture}

Myelogenous leukemia cell lines K562 and HL60 ${ }^{31}$ were cultured in flasks with DMEM medium containing $10 \%$ FBS and $1 \%$ penicillin-streptomycin, and were kept in a $5 \% \mathrm{CO}_{2}$ incubator (Bionex@, Korea) at $37{ }^{\circ} \mathrm{C}$ for regular culture. Cells for indentation tests were cultured inside fibronetin pre-coated confocal dishes for 72 hours with the purpose of sticking the cells to a substrate.

\subsection{Leukocyte isolation}

Normal leukocytes were obtained from a healthy adult donor by ficoll ${ }^{\mathrm{TM}}$-Paque 
Plus reagent (GE Healthcare, USA) and were then directly cultured in a confocal dish for 4 hours for attaching onto a substrate. Then the dish containing the cells was placed in a mini-incubator (Okolab, Italy) integrated into the sample stage of the optical tweezers for cell indentation tests. Approval for this part of the work was obtained from the Human Research Ethics Committee for Non-Clinical Faculties of the University of Hong Kong (approval reference number: EA240913).

\subsection{Optical tweezers and force calibration}

The optical tweezers employed in the experiments is a MMI Cell Manipulator System (MMI, Switzerland) with a maximum trapping power of $8 \mathrm{~W}$ (Nd:YAG laser, wavelength $1064 \mathrm{~nm}$ ). The $\mathrm{CO}_{2}$ mini-incubator setup on the microscope stage of the optical tweezers provides a good culture condition for the tested cells to survive during experiments. The microscope stage is controlled by the MMI software, which allows the position of the tested cell to be well controlled. The laser focus was pre-calibrated following instructions of the manual of the MMI Cell Manipulator.

The movement of the trapped bead was detected with a photodiode sensor integrated in the system. The relationship between the changes of the detected laser signal $\Delta D_{x}$ and $\Delta D_{y}$ along directions $x$ and $y$ respectively, and the movement $\Delta x$, $\Delta y$ of the bead, is given as $\Delta x=A \Delta D_{x}$ and $\Delta y=A \Delta D_{y}$, where $A$ is a sensitivity factor. The force measurement procedure used followed the harmonic oscillation method of Anderson, et al ${ }^{22}$, which is based upon the dynamics equation $F(x, t)=6 \pi \eta \alpha \dot{x}$ for a micrometer sized particle moving in a Newtonian liquid, where $F(x, t), \alpha$ and $\eta$ are the external particle force, particle radius and the dynamic viscosity, respectively. The trap force behaves as a harmonic spring which follows Hooke's law $F=k x$. When the particle is oscillated, a phase lag $\theta$ due to the viscous drag exists between the trap and the particle. The trap stiffness was obtained from the 
measured $\theta$ as $k=\frac{6 \pi \eta \omega \omega}{\tan \theta}$ at a particular oscillating frequency $\omega$. The laser trap stiffness versus laser power relation was determined by using a series of increasing laser powers to oscillate a particle in liquid. The sensitivity factor $A$ of the photodiode was also calibrated beforehand by the following procedure. A microsphere was first trapped at a marker position from which the reflected path of the laser would hit close to the center of the photodiode detector, as previously aligned by the supplier of the machine. The voltage signal detected by the photodiode at this point was then offset to zero. After that, the microsphere was oscillated slightly along the $x$ or $y$ direction, and the sensitivity for this direction was calibrated as the slope of the linear fit of the recorded movement versus the photodiode signal.

\subsection{Indentation procedure}

The confocal dish cultured with the tested samples was put into the mini-incubator integrated into the optical tweezers system. Polystyrene microspheres with diameter $2.49 \pm 0.02 \mu \mathrm{m}$ (Polysciences, USA) were added into the dish, with the sphere concentration pre-tested to avoid being too low or too high for testing the cells in a convenient manner. The above force calibration procedure was performed in the cell medium. Many leukemia cells and normal leukocytes were found to attach to the glass substrate firmly since the latter was pre-coated with fibronetins and collagens.

When performing lateral indentation on the cells, a laser power of $0.8 \mathrm{~W}$ was used. The indentation procedure used was as follows. First, the laser was used to trap one suspending sphere as seen at the center of the microscope screen by adjusting the $x-y$ stage. Then, the $x-y$ stage was moved to bring a targeted cell to approach one side of the sphere without solid cell-sphere contact. Finally, the stage movement was controlled to follow an indentation scheme of extension, hold and retraction, as shown schematically in figures $1 \mathrm{~A}$ and $\mathrm{B}$. To ensure that the trapped bead was indented on the cell at its maximum diameter plane as shown in figure $1 \mathrm{~A}$, the trapping objective and the detecting objecting of the optical trap system were well adjusted. This was 
done by first adjusting the vertical position of the specimen table so that the maximum diameter plane of the cell to be indented was brought into focus under the optical microscope. Then, the Z-axis focus of the laser beam was adjusted to bring the trapped bead to the same height as the maximum diameter plane of the cell with the purpose to let the indentation location would always be at the maximum diameter plane of the cell.

K562 cells were used to test the effect of loading rate on cell stiffness by the Hertzian and rate-jump methods. The indentation speed for the Hertzian method were set to be $0.078 \mu \mathrm{m} / \mathrm{s}, 0.156 \mu \mathrm{m} / \mathrm{s}$ or $0.312 \mu \mathrm{m} / \mathrm{s}$ with a total displacement of $0.78 \mu \mathrm{m}$, followed by a $10 \mathrm{~s}$ hold and then retraction at speed of $0.078 \mu \mathrm{m} / \mathrm{s}$. In the rate-jump method, the initial indentation speed was $0.078 \mu \mathrm{m} / \mathrm{s}$ to a displacement of $0.78 \mu \mathrm{m}$, followed by a $10 \mathrm{~s}$ hold, and then retraction at speed of $0.078 \mu \mathrm{m} / \mathrm{s}, 0.156 \mu \mathrm{m} / \mathrm{s}$ or $0.312 \mu \mathrm{m} / \mathrm{s}$. it should be noted that the indentation speed here is actually the speed at which the trapped bead be brought by the laser into contact with the cell in the liquid medium, and select an appropriate indentation speed here is quite critical: it cannot be too fast, otherwise the trapped bead can be lost before it contact to the cell due to the large viscos drag force, and also it may result in insufficient data to be collected for the further analysis. In this case, the lowest speed $0.078 \mu \mathrm{m} / \mathrm{s}$ adopted here is actually the minimum movement speed of the sample stage, the other two loading rate $0.156 \mu \mathrm{m} / \mathrm{s}$ or $0.312 \mu \mathrm{m} / \mathrm{s}$ is actually the twice and 4 times of this minimum value so that enable the indentation at various loading rate to be performed. Also, instantaneous retraction was not used in the present experiments because the acceleration of the bead involved is often too large during a sudden retraction so that it may break loose from the laser trap easily.

The nucleus of a cell is likely to be harder and so its presence inside a cell may affect the measured modulus. To gain understanding of this, a high laser power of 1.6 watts was used to incident on the rim of a K562 cell to rupture its membrane ${ }^{32}$. After waiting for certain time to let the membrane degrade and the cytoplasm flow away, lateral indentation on the exposed cell nucleus was performed. 


\subsection{Calculation of elastic modulus}

In the optical tweezers system, a position-sensitive photodiode is used to detect the displacement of the trapped bead from the center of the laser beam, as shown in Figure 1A. Before the cell makes contact with the bead, the initial photodiode signal $D_{0}$ is constant, since the bead is trapped by the laser beam at its center, and the only force applied on the bead is that due to Brownian motion, which is very small and can be neglected. Once the cell makes contact with the trapped bead, the bead is displaced and the photodiode signal will change to a new value $D$, and we have following relationships:

$h=Z-d$

$d=A\left(D-D_{0}\right)$

$F=k d=A\left(D-D_{0}\right)$

where $Z$ is the movement of the specimen stage after the initial cell-bead contact, $d$ is the movement of bead from center of the laser beam, $h$ is the indentation depth of the bead into the cell, $S$ is the photodiode sensitivity, and $k$ is the spring constant of the laser trap (see Fig. 1A). With eqn. (3), the indentation force $F$ on the cell can be known, so that the $Z-D$ curve recorded by the machine can be converted to an $F-h$ (indentation force vs depth) curve.

For the rate-jump method, by analyzing the force- and depth-rate changes before and after the unloading point, the elastic stiffness $S_{\mathrm{e}}$ of the cell-bead contact is given as $[12,13]$

$$
S_{e}=\frac{\dot{F}_{u}-\dot{F}_{h}}{\dot{h}_{u}-\dot{h}_{h}}
$$


where $\dot{F}_{u}, \dot{F}_{h}, \dot{h}_{u}, \dot{h}_{h}$ are the force rate just after unload, the force rate just before unload, the depth rate just after unload and the depth rate just before unload respectively.

The $2.5 \mu \mathrm{m}$ microspheres used as the indenters in the present experiments were much smaller than all the tested sample cells. For this reason, the indentation problem can be regarded as a classical problem of spherical indentation on a flat half-space, for which the contact stiffness is given as 33,34

$S_{e}=2 E_{r} a$

Here $a$ is the contact radius at full load (i.e. the onset of unload), which is given by

$a=\sqrt{R h_{u}}$

where, $R$ is the radius of the sphere, and $h_{u}$ is the depth just after unload. The reduced modulus $E_{r}$ can therefore be obtained from eqns. (4) to (6) as

$$
E_{r}=\frac{\dot{F}_{u}-\dot{F}_{h}}{2\left(\dot{h}_{u}-\dot{h}_{h}\right) \sqrt{R h_{u}}}
$$

The elastic modulus of the cell can then be obtained by

$$
\frac{1}{E_{r}}=\left(\frac{1-v^{2}}{E}\right)_{\text {sample }}+\left(\frac{1-v^{2}}{E}\right)_{\text {indenter }}
$$

where $v$ is the Poisson ratio and $E$ is Young modulus. Since $E$ is $3 \sim 3.6 \mathrm{GPa}$ for polystyrene while that for the cell is in the Pa range, $\left(1-v^{2} / E\right)$ for the indenter is much 
smaller than that for the cell and can be neglected, i.e. $E_{r} \approx\left[E /\left(1-v^{2}\right)\right]_{\text {sample }}$ is accurate enough. The $E$ value for the cell can therefore be calculated from the measured $E_{r}$ value. In this work, the Poisson ratio $v$ for the cell is taken to be $0.5^{35,36}$.

In the Hertzian method, the $F-h$ data are fitted to the relation ${ }^{29}$

$$
F=\frac{4}{3} E_{r} \sqrt{R} h^{3 / 2}
$$

in order to obtain the reduced modulus $E_{r}$.

\subsection{Statistics}

Tests were performed on certain randomly selected cells for each group. The average and standard deviation of the elastic modulus of each group of the samples tested by the Hertzian protocol were calculated. Two-population $t$-tests were performed on the rate-jump tested samples, where a significance level $p$ value less than 0.05 indicates significant difference between the tested groups.

\section{Results and Discussion}

The laser-trap-stiffness-vs-laser-power calibration was performed in the cell culture medium and the result exhibits a linear relationship when the laser power is lower than 1.6 watts, as shown in figure 2. When the laser power is higher than 1.6 watts, the trend of stiffness versus power becomes nonlinear. In our experiments, a power of 0.8 watt was used. Non-adherent cells such as leukocytes and leukemia cells are among the softest living cell types, and so their stiffness is even more difficult to be measured accurately. In vitro culture with pre-coated proteins such as fibronetin or collagen on a substrate enables such cells to be handled in this work. Some cells such as K562, macrophage and/or certain granulocytes do not even need such protein fixing. We have shown that optical tweezers can be used to perform indentation on these cells ${ }^{20}$. 
Here we compare the measurement of cell stiffness by the Hertzian and rate-jump methods. In figure 3, the K562 cell line was used as prototype for the Hertzian protocol, with loading rates $0.078 \mu \mathrm{m} / \mathrm{s}, 0.156 \mu \mathrm{m} / \mathrm{s}$ and $0.312 \mu \mathrm{m} / \mathrm{s}$. Figure 3A shows that the force vs. displacement curve shifts upward with loading rate. The apparent elastic moduli of the cells, calculated by fitting eqn. (9) to the force-depth curves in the 0 to $0.4 \mu \mathrm{m}$ depth range, with standard deviation from 12 samples are shown in figure $3 \mathrm{~B}$; it can be seen that the measured modulus increases with loading rate. Two-population $t$ tests performed for all groups return $p$ values all lower than 0.01 , indicating that the values at different loading rates are significantly different. The increment of the apparent modulus with loading rate is a typical response of viscoelastic materials ${ }^{37}$. Figure 4 shows the same cell type tested by the rate-jump method. Figure 4A and 4B show typical depth-time and force-time curves measured during indentation on the softest part of K562 cells at different unloading rates respectively, from which the elastic modulus is extracted out at the onset of unload. Although the total displacement of the sample stage towards the trapped bead was $0.78 \mu \mathrm{m}$, this comprised the stage movement before bead-cell contact, the deflection of the trapped bead and the indentation depth of the bead into the cell. Therefore, the maximum indentation depth as seen in Figure 4B falls short of $0.78 \mu \mathrm{m}$. In Figure 4A, on initial contact during, say, the first 5 to 7 seconds, the indentation force was nearly zero or negative, and it is only when the indentation depth became larger that the indentation force turned into positive. This suggests a small adhesive effect between the cell and the indenter bead on initial contact. Figure $4 \mathrm{C}$ is the resultant elastic modulus at different unloading rates calculated from eqns. (7) and (8). Two-population $t$ tests for each pair of unloading-rate groups return $p$ values all higher than 0.05 , indicating that the elastic moduli at the three unloading rates are not significantly different. The results here show that the rate-jump method can yield elastic modulus independent of the test condition (i.e. the unloading rate), and so it is a reliable method for measuring the intrinsic elastic modulus of non-adherent cells.

Figure 5 shows the test results of leukemia cells and normal leukocytes by the 
rate-jump method, as well as those of the nuclei of K562 cells. Figure 5A and 5B show the force-time curve and depth-time curves of all cell types tested. The elastic modulus of each group is summarized in Figure 5C, which shows that the nuclei of K562 have the highest elastic modulus of all groups. The measured average elastic moduli of K562 and HL60 cells are 74.62 and $90.33 \mathrm{~Pa}$ respectively, which are obviously higher than the values of macrophage, monocyte and granulocyte, which are 41.45, 30.77 and $22.36 \mathrm{~Pa}$, respectively. The measured modulus of K562 nucleus is nearly twofold of the K562, which verifies the hypothesis that the cell nucleus is stiffer than the cell as a whole. This is also consistent with earlier findings ${ }^{38}$.

It is well known that it is hard to extract the intrinsic elastic modulus of cells, since their overall force-deformation response is an overall effect of their viscoelastic or poro-viscoelastic behaviour. For instance, if the conventional Herztian method is used, large variations in the result may arise simply because different loading rates are used, as illustrated in Figure 3B. The rate-jump method employed in this work was designed to overcome this difficulty ${ }^{12}$, since for any sample considered as viscoelastic, the viscous influence is nullified by a sudden rate jump in the loading, such as that at the onset of the retraction. This method has been demonstrated to be valid on many viscoelastic materials such as soft metals ${ }^{39}$, gels ${ }^{40}$, and adherent cells ${ }^{9}$. This is the first time the validity of the method is demonstrated for non-adherent cells with extremely low measurement forces in the pico-Newton regime. Also, this is the first time the method is adapted for use in an optical tweezers system. Figure 4 shows that, when compared to the Hertzian protocol, the rate-jump method can effectively give invariant and reliable elastic modulus of very soft non-adherent cells.

Living cells are normally considered as dynamic and whenever reorganization of cytoskeleton takes place, the cell stiffness should change as the cytoskeleton changes. The cell geometry affects adherent cells significantly ${ }^{41}$. Yet, for non-adherent cells, such a concern becomes unimportant since their shapes remain nearly spherical most of the time under in vivo environments. Regarding whether diseased leukemia cells or 
their normal counterparts are stiffer, the elastic modulus is not only affected by the cytoskeleton but also by the nucleus-to-cytoplasm ratio of the cell ${ }^{25,42}$, Most of the cancerous cells are known to have a higher nucleus-to-cytoplasm ratio, and since the elastic modulus of the nucleus is higher than that of the cell as a whole, a higher measured elastic modulus is expected as is the case in the present experiments.

One should also note that normal leukocytes include a mixture of cell types, but chemical staining to identify certain cell types was not done due to the need to perform single cell manipulation in living conditions in the present experiments. Thus, only three cell types that could be identified under the optical microscope, including macrophage, monocyte and granulocyte, were measured, and also only the spherical macrophages were selected to be measured since their morphology would change as they move around in a liquid medium. The measured elastic moduli of these normal leukocytes and leukemia cells from the rate-jump method in Figure 5 are consistent with the hypothesis that leukostasis ${ }^{43}$ caused by leukemia cells may be closely related to their high stiffness ${ }^{44,45}$, since large leukemia cells with high stiffness may easily block microcapillaries, and cause the segregation of cells in certain organs, namely, leukostasis. Interestingly, the elastic moduli of cells measured with the rate-jump method agree with the expectation that in a healthy person, leukostatsis does not happen. However, for patients suffering from leukemia, the risk of leukostasis is larger in acute myelogenous leukemia than chronic myelogenous leukemia. For this reason, an accurate evaluation and understanding of the stiffness of cancerous non-adherent cells is important in understanding the mechanism of leukostasis.

The use of optical tweezers for the nanomechanical characterization of cells as stipulated in this work has a distinctive advantage over depth-sensing or AFM nanoindentation. In the present approach, the indenter probes are microspheres originally suspending freely in the culture medium for the cells, inside a microscope incubator integrated into the optical tweezers system. Thus, suitable culture conditions, e.g. for the cells to reach a certain life-cycle stage, can be conveniently maintained for 
as long as needed, before the laser is switched on to trap a nearby bead to do indentation on any chosen cell. In this case, disturbance arising from the transfer of samples from an external incubator to the test platform, or from hydrodynamic effects involved in the massive movement of an indenter probe physically connected to the machine, as in the case of an AFM or a commercial nanoindenter, can be eliminated.

The rate-jump optical-tweezers indentation method proposed in this work is a local measurement method which can yield intrinsic modulus (figure 4) as well as resolve the small differences in stiffness between very soft cell types and their nuclei (figure 5). This method should therefore be useful to study the relation between disease malignancy and the mechanical properties and substructure response of cells of various clinical conditions. Also, the proposed method enables the intrinsic elastic modulus of cells to be accurately measured, and so it should enable the effects on mechanical properties due to reorganization of the cytoskeleton network ${ }^{46}$ during cell adhesion ${ }^{47}$, maturation ${ }^{48}$ and differentiation ${ }^{47,49}$ to be investigated. The change of cell shape, motility, mitosis and the anchorage-dependent growth is known to be tightly linked to the whole cell structure ${ }^{50}$. Many unknown stiffness relations involved in the events may also be able to be explored by the proposed method, in order to identify their roles in the cell growth, locomotion, migration and metastasis. Furthermore, fewer studies have been made on nuclear mechanics compared to the mechanics of cytoskeleton network ${ }^{51}$, although the mechanical properties of the nucleus are closely involved in the expression of nuclear components, modulation of cytoskeletal network and participation of cellular functions. The difference of the nuclear structure and composition is also relevant to many human diseases ${ }^{51}$, and our current results on the nucleus mechanics of K562 cells can serve as a good start for in-depth research on nuclear mechanotransduction ${ }^{52}$, cell signaling and gene transcription ${ }^{52,53}$. 


\section{Conclusions}

A rate-jump method for elastic modulus measurement was implemented in an optical tweezers system for microsphere indentation experiments on non-adherent cells. Even for cells involving indentation forces as small as in the pico-newton regime, this method yields intrinsic elastic moduli that are invariant with respect to the test condition, namely, the magnitude of the rate jump. This method is capable of grading the stiffness of different cell types, including K562 and HL60 cell lines and normal leukocytes. The nucleus of K562 is also found to be significantly stiffer than the cell as a whole. The present rate-jump indentation method with optical tweezers can serve as an assay for the accurate measurement of the stiffness of soft non-adherent cells.

\section{Acknowledgement}

The authors acknowledge the support of a Special Equipment Grant no. SEF-HKU06 from the University Grants Committee of the Hong Kong SAR Government, and a Project 11202039 supported NSFC, as well as a basic research

fund from the Shenzhen Government (Project Number 201208303000415), P.R. China. 


\section{References}

1. W. A. Lam, M. J. Rosenbluth and D. A. Fletcher, Blood, 2007, 109, 3505-3508.

2. C. Zou, Q. Luo, J. Qin, Y. Shi, L. Yang, B. Ju and G. Song, Cell Biochem Biophys, 2013, 65, 455-462.

3. A. Pillarisetti, J. P. Desai, H. Ladjal, A. Schiffmacher, A. Ferreira and C. L. Keefer, Cellular reprogramming, 2011, 13, 371-380.

4. Q. S. Li, G. Y. Lee, C. N. Ong and C. T. Lim, Biochem Biophys Res Commun, 2008, 374, 609-613.

5. Z. Zhou, C. Zheng, S. Li, X. Zhou, Z. Liu, Q. He, N. Zhang, A. Ngan, B. Tang and A. Wang, Nanomedicine, 2013, 9, 864-874.

6. R. M. Hochmuth, J Biomech, 2000, 33, 15-22.

7. A. R. Bausch, F. Ziemann, A. A. Boulbitch, K. Jacobson and E. Sackmann, Biophys J, 1998, 75, 2038-2049.

8. A. Chabanel, K. L. Sung, J. Rapiejko, J. T. Prchal, J. Palek, S. C. Liu and S. Chien, Blood, 1989, 73, 592-595.

9. Z. L. Zhou, A. H. Ngan, B. Tang and A. X. Wang, J Mech Behav Biomed Mater, 2012, 8, 134-142.

10. N. Desprat, A. Richert, J. Simeon and A. Asnacios, Biophys J, 2005, 88, 2224-2233.

11. F. Wottawah, S. Schinkinger, B. Lincoln, R. Ananthakrishnan, M. Romeyke, J. Guck and J. Kas, Phys Rev Lett, 2005, 94, 098103.

12. A. H. W. Ngan and T. Bin, J. Mater. Res, 2009, 24, 10.

13. T. B and N. A. H. W, Soft Matter, 2012, 8, 6 .

14. Y. L. Chan and A. H. W. Ngan, Polymer Testing, 2010, 29, 7.

15. A. C. Fischer-Cripps, Materials Science and Engineering a-Structural Materials Properties Microstructure and Processing, 2004, 385, 74-82.

16. A. E. Giannakopoulos, Journal of the Mechanics and Physics of Solids, 2006, 54, 1305-1332.

17. M. V. R. Kumar and R. Narasimhan, Current Science, 2004, 87, 1088-1095.

18. H. Lu, B. Wang, J. Ma, G. Huang and H. Viswanathan, Mechanics of Time-Dependent Materials, 2003, 7, 189-207.

19. M. Vandamme and F. J. Ulm, International Journal of Solids and Structures, 2006, 43, 3142-3165.

20. Z. L. Zhou, B. Tang and A. H. W. Ngan, Nano Life, 2012, 2, 1-10.

21. Z. L. Zhou, B. Tang, A. H. W. Ngan, Z. N. Dong and Y. S. Wu, let Nanobiotechnol, 2012, 6, 9-15.

22. M. Andersson, A. Madgavkar, M. Stjerndahl, Y. R. Wu, W. H. Tan, R. Duran, S. Niehren, K. Mustafa, K. Arvidson and A. Wennerberg, Rev Sci Instrum, 2007, 78.

23. X. Cai, X. Xing, J. Cai, Q. Chen, S. Wu and F. Huang, Micron, 2010, 41, 257-262.

24. D. S. Gokhin, R. B. Nowak, N. E. Kim, E. E. Arnett, A. C. Chen, R. L. Sah, J. I. Clark and V. M. Fowler, Plos One, 2012, 7, e48734.

25. Z. L. Zhou, C. X. Zheng, S. Li, X. F. Zhou, Z. H. Liu, Q. T. He, N. N. Zhang, A. H. W. Ngan, B. Tang and A. X. Wang, Nanomedicine : nanotechnology, biology, and medicine, 2013, (DOI 10.1016/j.nano.2013.1004.1001).

26. S. Sen, S. Subramanian and D. E. Discher, Biophys J, 2005, 89, 3203-3213.

27. T. P. Prevost, A. Balakrishnan, S. Suresh and S. Socrate, Acta biomaterialia, 2011, 7, 83-95. 
28. H. Byun, T. R. Hillman, J. M. Higgins, M. Diez-Silva, Z. Peng, M. Dao, R. R. Dasari, S. Suresh and Y. Park, Acta biomaterialia, 2012, 8, 4130-4138.

29. H. P. Koeffler and D. W. Golde, Blood, 1980, 56, 344-350.

30. H. Hertz, 1882.. and . 92, J. Reine Angew. Math, 1882, 92, 16.

31. R. Gallagher, S. Collins, J. Trujillo, K. McCredie, M. Ahearn, S. Tsai, R. Metzgar, G. Aulakh, R. Ting, F. Ruscetti and R. Gallo, Blood, 1979, 54, 713-733.

32. K. S. Ng, Z. L. Zhou and A. H. W. Ngan, J Cell Physiology, 2013, 228, 2037-2041.

33. K. L. Johnson, Contact Mechanics, Cambridge University Press, Cambridge,UK, 1985.

34. I. N. Sneddon, Int. J. Eng. Sci., 1965, 3.

35. M. J. Rosenbluth, W. A. Lam and D. A. Fletcher, Biophysical Journal, 2006, 90, 2994-3003.

36. E. J. Koay, A. C. Shieh and K. A. Athanasiou, Trans. ASME, J. Biomech. Eng., 2003, 125, 334-341.

37. K. KE, R. AC, L. J, A. TE, B. CP, K. GH and W. DA, Curr Opin Cell Biol, 2007, 19, 7.

38. F. Guilak, J. R. Tedrow and R. Burgkart, Biochem Biophys Res Commun, 2000, 269, 781-786.

39. A. H. W. Ngan and T. Bin, J. Mater. Res. , 2002, 17, 7.

40. B. Tang and A. H. W. Ngan, J. Mater. Res., 2003, 8, 8.

41. S. Y. Tee, J. Fu, C. S. Chen and P. A. Janmey, Biophys J, 2011, 100, L25-27.

42. F. H. White, Y. Jin and L. Yang, Histology and histopathology, 1997, 12, 69-77.

43. J. M. Rowe and M. A. Lichtman, Blood, 1984, 63, 1230-1234.

44. M. A. van Buchem, P. C. Hogendoorn, C. N. Levelt, P. van Hengel, L. P. Colly, P. M. Kluin and R. Willemze, Leukemia, 1992, 6, 142-149.

45. W. A. Lam, M. J. Rosenbluth and D. A. Fletcher, British journal of haematology, 2008, 142, 497-501.

46. M. F. Leung, J. A. Sokoloski and A. C. Sartorelli, Cancer Res, 1992, 52, 949-954.

47. F. Lautenschlager, S. Paschke, S. Schinkinger, A. Bruel, M. Beil and J. Guck, Proc Natl Acad Sci U S A, 2009, 106, 15696-15701.

48. H. Takano and K. Abe, Methods in molecular biology, 2004, 242, 85-94.

49. B. Stadelmann, E. Khandjian, A. Hirt, A. Luthy, R. Weil and H. P. Wagner, Leuk Res, 1990, 14, 815-821.

50. S. D. Bernal and R. A. Stahel, Critical reviews in oncology/hematology, 1985, 3, 191-204.

51. M. Zwerger, C. Y. Ho and J. Lammerding, Annual review of biomedical engineering, 2011, 13, 397-428.

52. K. N. Dahl, A. J. Ribeiro and J. Lammerding, Circ Res, 2008, 102, 1307-1318.

53. A. J. Ribeiro, S. Tottey, R. W. Taylor, R. Bise, T. Kanade, S. F. Badylak and K. N. Dahl, J Biomech, 2012, 45, 1280-1287. 


\section{Figures}

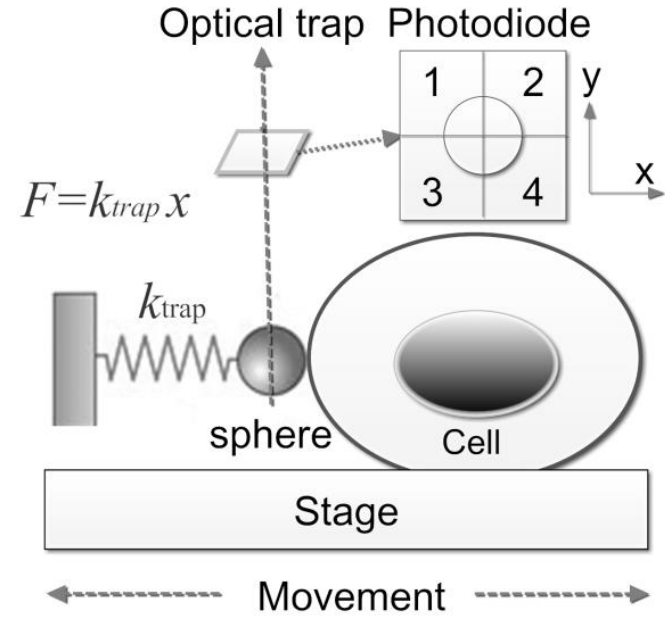

A

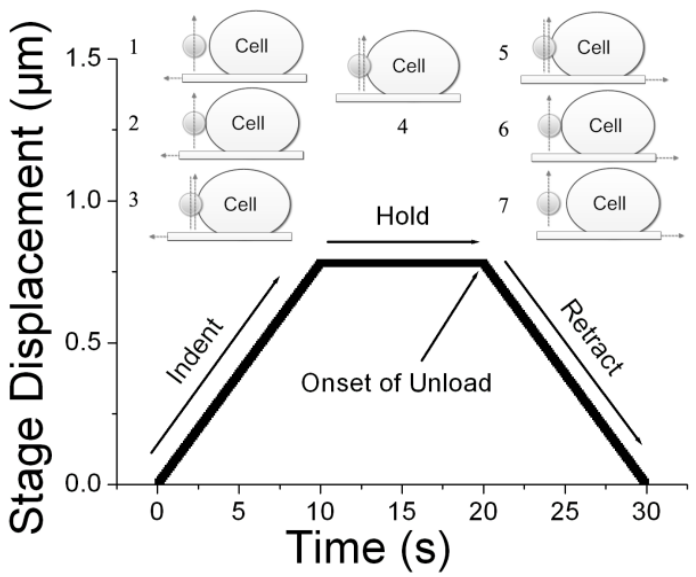

B

Figure 1. A: Schematic showing cell indentation by optical tweezers. The position of the sphere on the $\mathrm{x}-\mathrm{y}$ plane perpendicular to the laser beam is monitored by a photodiode as shown. B: Stage displacement vs. time curve used in the indentation tests. The indentation of all samples was carried out to a peak displacement of $0.78 \mu \mathrm{m}$, followed by 10 second hold and 10 second retraction. The inset graphs show the corresponding process of the indentation on the cell by sphere. Inset images 1, 2 and 3 show the indentation process, image 4 holding and images 4,5 and 6 the retraction process. 


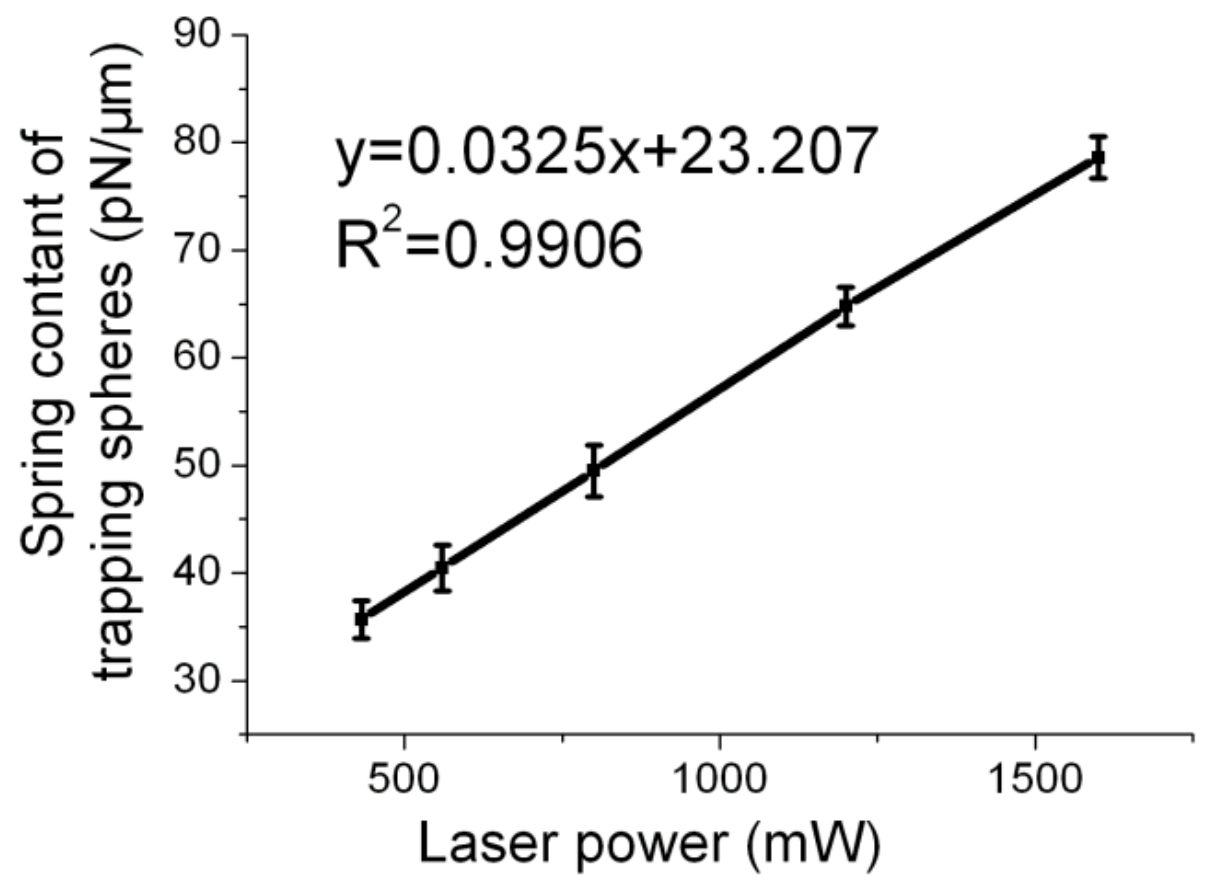

Figure 2: Spring constant of optical trap on $2.5 \mu \mathrm{m}$ polystyrene spheres at different laser power. 


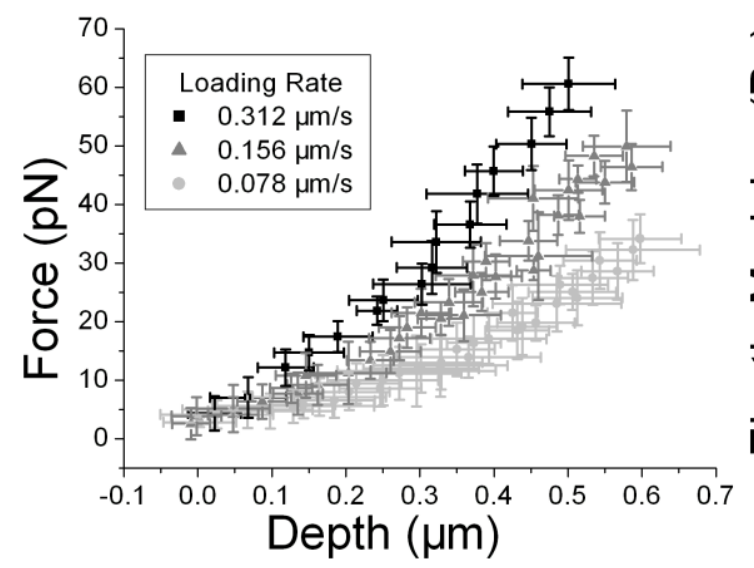

A

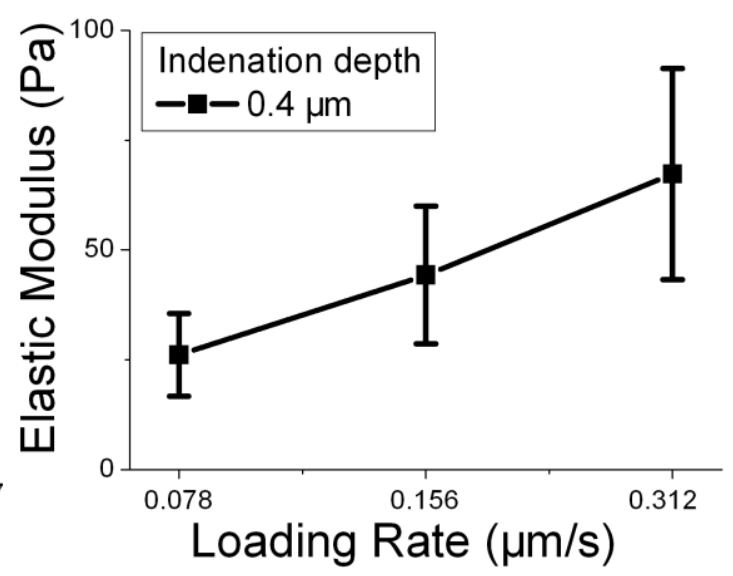

$\mathrm{B}$

Figure 3. Elastic modulus of K562 cells measured using the Hertzian method. A: Representative curves of force versus indentation depth at different loading rates; B: The average values of elastic modulus with standard deviation measured from 12 samples at different loading rates. 

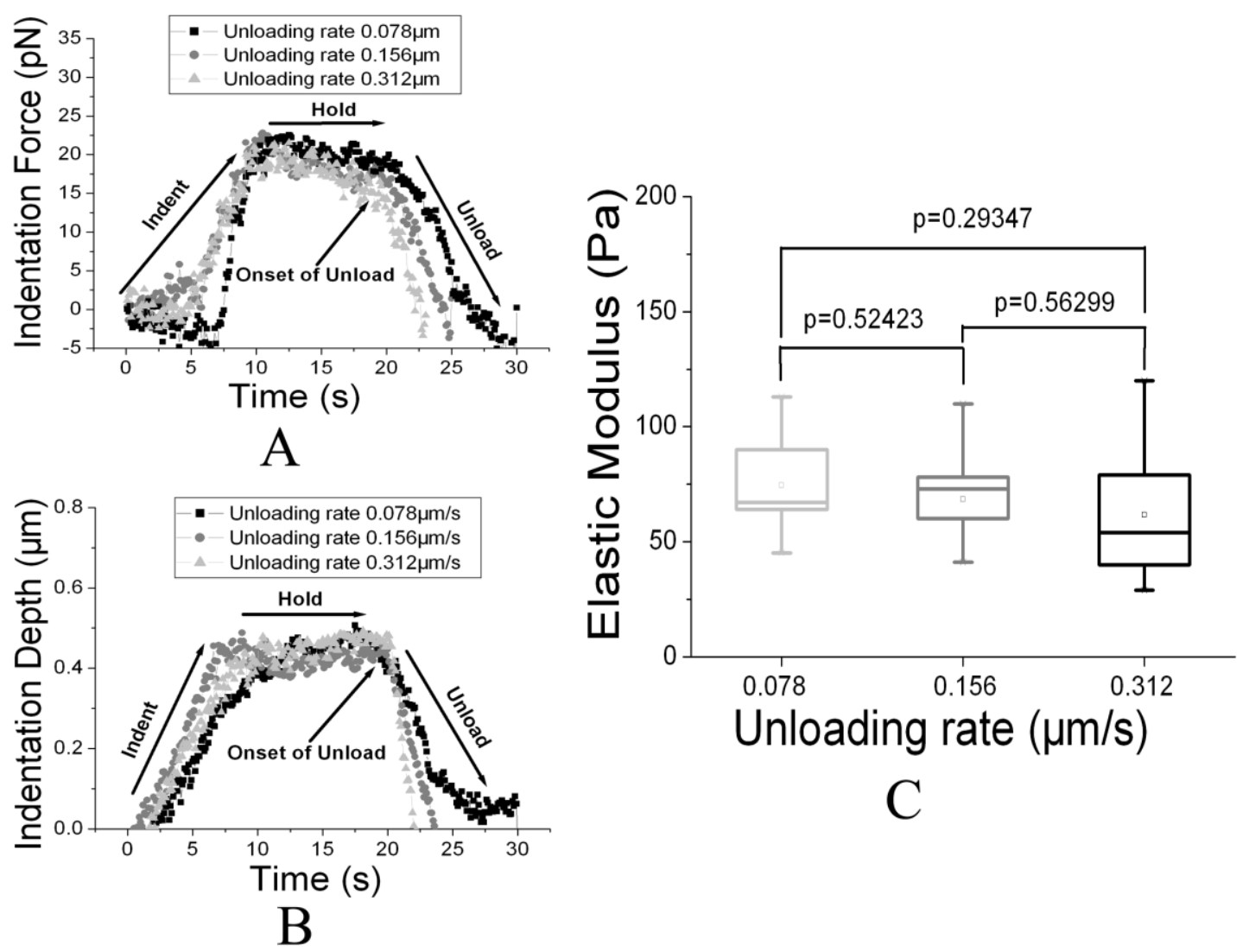

Figure 4. Results of K562 cell indentation by the rate-jump method. A: Indentation force $P$ vs. time in rate-jump tests using unloading rates of $0.078 \mu \mathrm{m} / \mathrm{s}, 0.156 \mu \mathrm{m} / \mathrm{s}$ and $0.312 \mu \mathrm{m} / \mathrm{s}$; B. The corresponding indentation depth $h$ vs. time; C. Measured elastic modulus with different retraction rates. 12 samples at each unloading rate were tested. The box charts shown in figure 4C show data from each group. Two-population $t$ tests were performed in each pair of groups at different unloading rates. 


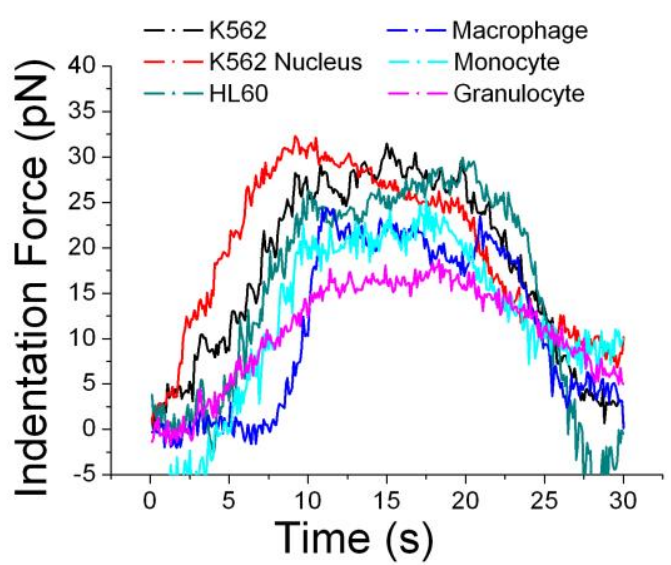

A

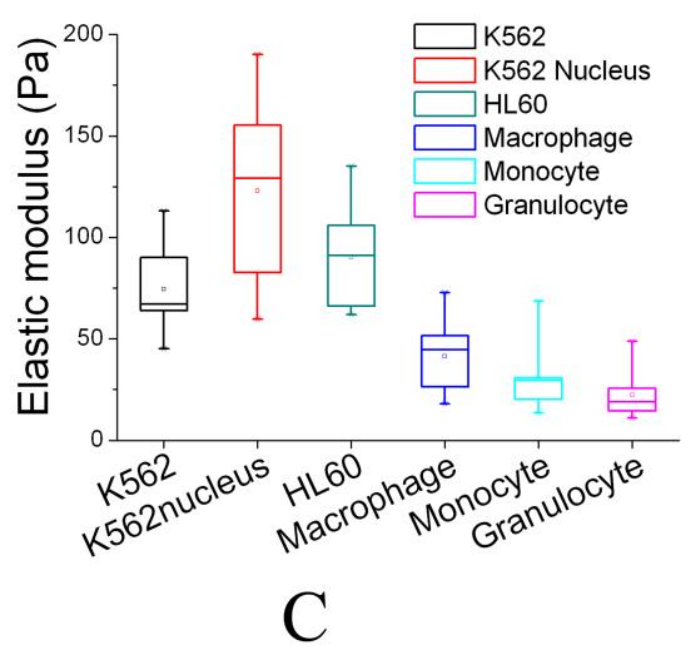

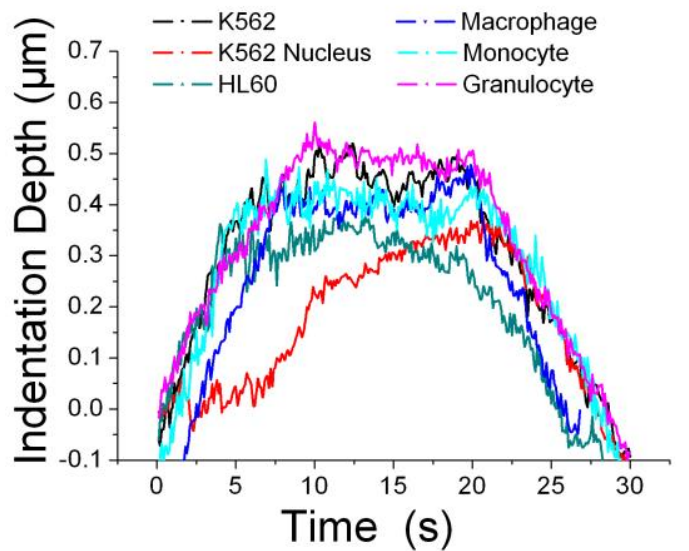

B

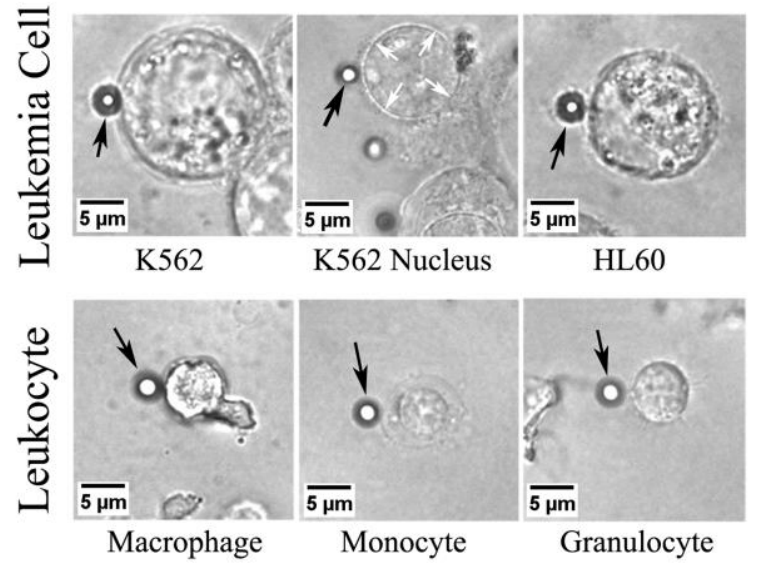

$\mathrm{D}$

Figure 5. Results of cell stiffness. A: Indentation force vs time corresponding to cells in figure 5D; B: The corresponding indentation depth vs time; C. Elastic moduli of all cell types, 12 samples were tested in each group except that 6 samples were tested on K562 nucleus; D: Indentation images of normal leukocytes and Leukemia cells. In all subfigures, the black arrows indicate the indenter sphere. In the case of K562 nucleus, the white arrows point at the circumferential membrane of the nucleus under indentation, indicating the position of the indented nucleus. 